\title{
Assessment Of Quickbird High Spatial Resolution Imagery To Detect Red-Attack Damage Due To Mountain Pine Beetle Infestation
}

Nicholas C. Coops ${ }^{1 *}$, Matt Johnson ${ }^{1}$, Michael A. Wulder ${ }^{2}$, Joanne C. White ${ }^{2}$

1-Department of Forest Resource Management, 2424 Main Mall. University of British Columbia, Vancouver. Canada. V6T 1 Z4

2-Canadian Forest Service (Pacific Forestry Centre),

Natural Resources Canada, Victoria, British Columbia, Canada, V8Z 1M5

${ }^{*}$ Corresponding author:

Nicholas Coops

Phone: (604) 822 6452, Fax (604) 822-9106, Email: Nicholas.coops@ubc.ca

Pre-print of published version.

Reference:

Coops, N.C., Johnson, M., Wulder, M.A., White, J.C., 2006. Assessment of QuickBird high spatial resolution imagery to detect red attack damage due to mountain pine beetle infestation. Remote Sensing of Environment. 103: 67-80

DOI:

doi:10.1016/j.rse.2006.03.012

\section{Disclaimer:}

The PDF document is a copy of the final version of this manuscript that was subsequently accepted by the journal for publication. The paper has been through

peer review, but it has not been subject to any additional copy-editing or journal specific formatting (so will look different from the final version of record, which may be accessed following the DOI above depending on your access situation). 


\section{ABSTRACT}

High spatial resolution remotely sensed data has the potential to complement existing forest health programs for both strategic planning over large areas, as well as for detailed and precise identification of tree crowns subject to stress and infestation. The area impacted by the current mountain pine beetle (Dendroctonus ponderosae Hopkins) outbreak in British Columbia, Canada, has increased 40 -fold over the previous five years, with approximately 8.5 million hectares of forest infested in 2005 . As a result of the spatial extent and intensity of the outbreak, new technologies are being assessed to help detect, map, and monitor the damage caused by the beetle, and to inform mitigation of future beetle outbreaks. In this paper, we evaluate the capacity of high spatial resolution QuickBird multi-spectral imagery to detect mountain pine beetle red attack damage. ANOVA testing of individual spectral bands, as well as the Normalized Difference Vegetation Index (NDVI) and a ratio of red to green reflectance (Red-Green Index or RGI), indicated that the RGI was the most successful $(p<0.001)$ at separating non-attack crowns from red attack crowns. Based on this result, the RGI was subsequently used to develop a binary classification of red attack and non-attack pixels. The total number of QuickBird pixels classified as having red attack damage within a 50 $\mathrm{m}$ buffer of a known forest health survey point were compared to the number of red attack trees recorded at the time of the forest health survey. The relationship between the number of red attack pixels and observed red attack crowns was assessed using independent validation data and was found to be significant $\left(r^{2}=0.48, p<0.001\right.$, standard error $=2.8$ crowns). A comparison of the number of QuickBird pixels classified as red attack, and a broader scale index of mountain pine beetle red attack damage 
(Enhanced Wetness Difference Index, calculated from a time series of Landsat imagery), was significant $\left(r^{2}=0.61, p<0.001\right.$, standard error $=1.3$ crowns $)$. These results suggest that high spatial resolution imagery, in particular QuickBird satellite imagery, has a valuable role to play in identifying tree crowns with red attack damage. This information could subsequently be used to augment existing detailed forest health surveys, calibrate synoptic estimates of red attack damage generated from overview surveys and/or coarse scale remotely sensed data, and facilitate the generation of value-added information products, such as estimates of timber volume impacts at the forest stand level. 


\section{INTRODUCTION}

Throughout Canada, and worldwide, there are growing expectations by the public, government, and industry for more detailed and accurate information relating the health and condition of forests. This information, along with forest inventory data, contributes towards government policy development, national and international reporting obligations, and commercial decisions relating to forest investment, planning, harvesting, and sales (Stone and Coops 2004). Forest health monitoring can be undertaken using a variety of survey techniques (Table 1), depending on the information needs of the end user (Wulder et al., 2006c).

Coarse scale forest health overview surveys are typically conducted on an annual basis to provide a gross estimate of forest health conditions over large areas (e.g., provinces, states). Often these surveys fulfill strategic level information needs and encompass many different forest health concerns. Conversely, detailed surveys are conducted over smaller areas and target specific forest pests or diseases. These detailed surveys generally rely on large scale data with a high spatial resolution (e.g., aerial photography) or ground sampling, and the locations for these surveys are typically guided by reconnaissance level information collected from the overview surveys. The actual survey approach used by forestry organizations is influenced by their individual needs (government vs. forest industry), their capacity and resources to implement the survey and assessment process, and the nature of their license area (Stone and Coops 2004). 
Table 1: Types of surveys used in the assessment of insect-related damage associated with crowns (adapted from Stone and Coops 2004).

\begin{tabular}{|c|c|c|c|}
\hline & $\begin{array}{l}\text { TEMPORAL } \\
\text { RESOLUTION }\end{array}$ & $\begin{array}{c}\text { SPATIAL } \\
\text { RESOLUTION }\end{array}$ & $\begin{array}{l}\text { VARIABLES } \\
\text { ASSESSED }\end{array}$ \\
\hline \multicolumn{4}{|l|}{$\begin{array}{l}\text { OVERVIEW } \\
\text { SURVEY }\end{array}$} \\
\hline $\begin{array}{l}\text { Describes gross } \\
\text { area damaged, } \\
\text { general location of } \\
\text { the damage, and } \\
\text { trend in damage (if } \\
\text { collected for } \\
\text { multiple time } \\
\text { periods). } \\
\text { Information is used } \\
\text { to determine the } \\
\text { impact of damage } \\
\text { on productivity and } \\
\text { for adjusting the } \\
\text { allowable annual } \\
\text { cut and timber } \\
\text { supply forecasts. }\end{array}$ & $\begin{array}{l}\text { Often annually or } \\
\text { less frequently. } \\
\text { Monitoring is } \\
\text { achieved if the } \\
\text { same areas are re- } \\
\text { surveyed using } \\
\text { repeatable } \\
\text { methodologies. }\end{array}$ & $\begin{array}{l}\text { Extensive areas } \\
\text { surveyed. } \\
\text { Coarse level data is } \\
\text { used or information } \\
\text { is captured at coarse } \\
\text { scales }(e . g ., \\
1: 100,000 \text { or } \\
1: 250,000) . \\
\text { Often take the form } \\
\text { of aerial } \\
\text { reconnaissance } \\
\text { surveys. }\end{array}$ & $\begin{array}{l}\text { From aerial } \\
\text { reconnaissance, } \\
\text { sketch maps of } \\
\text { canopy damage for } \\
\text { strategic planning } \\
\text { and identifying areas } \\
\text { requiring survey that } \\
\text { is more intensive. }\end{array}$ \\
\hline
\end{tabular}

\section{DETAILED}

SURVEY

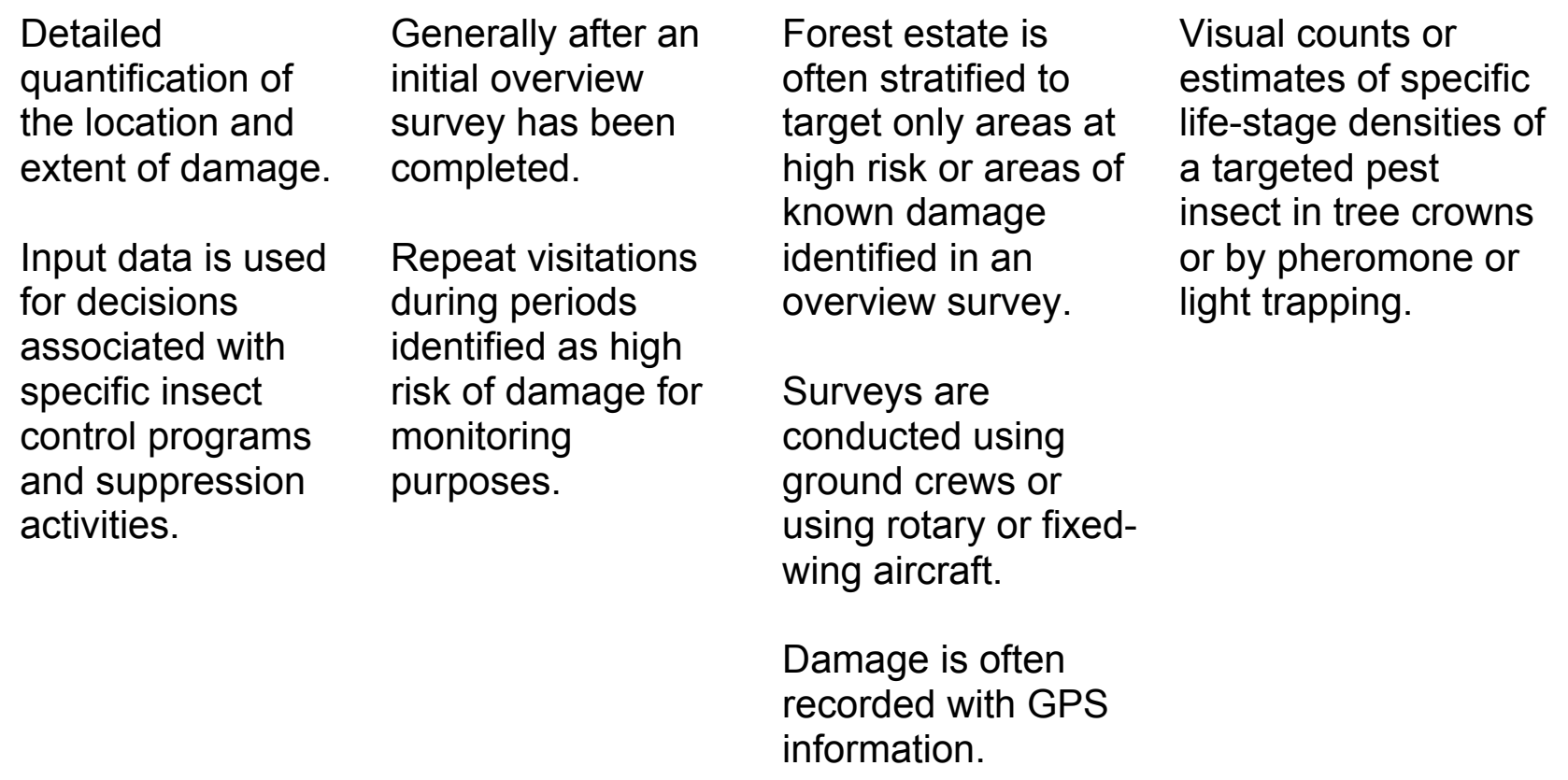


The mountain pine beetle (Dendroctonus ponderosae Hopkins) is one of the most destructive insects within western North America (Wood, 1963; Safranyik et al., 1974; Wood and Unger, 1996). The total area impacted by the current mountain pine beetle outbreak in British Columbia, Canada, increased from approximately 164,000 ha in 1999 to 8.5 million ha in 2005 (British Columbia Ministry of Forests and Range, 2005). The unprecedented extent of the damage resulting from the infestation has resulted in a need for information, with varying levels of detail, regarding the location and extent of beetle damage.

\subsection{Mountain Pine Beetle Biology}

The mountain pine beetle normally has an annual life cycle with four stages: egg, larva, pupa, and adult. In mid- to late-summer, female beetles attack the tree bole and lay their eggs beneath the bark. The eggs hatch shortly thereafter, and the resulting larva feed on the tree's phloem (Safranyik et al., 1974). By the following year, the larvae mature to adults, emerge from the tree, and spread to the next stand of susceptible host trees (Unger, 1993). The primary host, lodgepole pine (Pinus contorta), may experience extensive mortality when attacked. Following attack, the crown needles remain visibly unchanged; however, a drop in sapwood moisture leads to the cellular breakdown of needle structure (Murtha, 1978; Murtha and Wiart, 1987). 


\subsection{Host Spectral Response}

The physiological changes in the infested tree resulting from a mountain pine beetle attack manifest as changes in the color of the infested tree crown. Generally, the foliage fades from green to yellow to red over the spring and summer following attack (Amman, 1982; Henigman et al., 1999). Once the tree is killed, but still with green foliage, the host tree is in the green-attack stage. The first visible sign of impact is a change in foliage color from green to greenish-yellow that usually begins in the top of the crown (Ahern, 1988). These trees are referred to as faders. As the foliage fades from green to yellow to red over the spring and summer following attack, the foliage gradually desiccates and the pigments breakdown. This is known as the red attack stage. Initially the green chlorophyll pigments are lost, then the yellow carotenes and finally the red anthocyanins (Hill et al., 1967). At the red attack stage, infested trees exhibit significant chlorophyll degradation as the foliage dies and turns red (Murtha, 1978). Trees that have been dead for more than a year, and have shed the majority of their needles, are referred to as gray attack (Unger, 1993; British Columbia Ministry of Forests, 1995). The rate at which trees progress from green attack through red attack to gray attack is highly variable (Wulder et al., 2006a).

\subsection{Information Needs and Survey Methods}

The information needs of forest managers, in the context of addressing an infestation of mountain pine beetle, range from strategic planning over large areas, to detailed and precise locations for sanitation logging and individual tree treatments (Wulder et al., 2006c). Consequently, the scale of the information collected by forest health surveys 
ranges from broad (province-wide aerial overview sketch mapping), to more detailed (helicopter Global Positioning System (GPS) or heli-GPS surveys as they are commonly referred to), to detailed ground surveys for layout of blocks for sanitation logging and for fall and burn treatment.

Aerial overview surveys, which are typically conducted annually and encompass a wide variety of damage agents, provide sufficient information to characterize the general location of the damage, approximate the gross area of damage, and indicate general trends in damage from one year to the next (British Columbia Ministry of Forests and Canadian Forest Service, 2000). These broad surveys also provide an initial stratification of the landscape that can direct the collection of more detailed infestation information that will have greater spatial and attribute accuracy. Survey programs of this nature commenced for forest inventory in the late 1940's with widespread adoption in the 1950's (Frayer and Furnival, 1999). Aerial sketch mapping can be highly subjective with the accuracy of results being dependent on the knowledge, experience, and skill of the observer (McConnell et al., 2000; Aldrich et al., 1958; British Columbia Ministry of Forests and Canadian Forest Service, 2000); however, no other data source provides a comparable synoptic view for such a wide range of forest health issues, in a form that is rapidly available to forest managers.

In British Columbia, detailed aerial surveys, conducted mainly for the detection and mapping of bark beetles over smaller areas, are the responsibility of forest districts or licensees. These surveys, normally completed at a scale of 1:20,000 using a helicopter 
with a GPS receiver, record the centroid of individual infestation clusters. For each cluster, the number of infested trees is estimated and the damage agent is recorded. The size of the clusters may vary and the cluster area, shape, and compactness are not recorded (Nelson et al., 2004). The information collected from heli-GPS surveys is used primarily for expediting the deployment of field crews to find green attack in areas where suppression activities are recommended. An advantage of this survey method over others is the low error of commission: the surveyor is able to visually inspect each crown and can differentiate between different damage agents (e.g., porcupine girdling, flooding, mechanical damage, and bark beetles). The disadvantages of the heli-GPS surveys include the greater likelihood of omission error, resulting from the typical nonsystematic nature of the survey design, and the collection of damage information exclusive of data for healthy trees. Heli-GPS surveys are considered by the British Columbia Ministry of Forests to be the "operational benchmark for accuracy, delivery time and cost for detailed aerial surveys" and "the most commonly used detailed aerial survey method at this time"; heli-GPS locations are estimated to be spatially accurate to within $\pm 20 \mathrm{~m}$ (British Columbia Ministry of Forests, 2004). Nelson et al. (2006) assessed the accuracy of heli-GPS survey points with concurrently collected field data, and found that $92.6 \%$ of the heli-GPS survey points had errors of \pm 10 trees.

\subsection{Remotely Sensed Data for Red Attack Detection, Mapping, and Monitoring}

Digital satellite remote sensing offers a complementary technology for the detection and mapping of mountain pine beetle red attack damage with benefits that include the capacity to cover large spatial areas - ensuring a census, rather than a sample, of forest 
stands (Wulder et al., 2006a). In addition, remotely sensed data can be easily integrated with other spatial data (such as roads, elevation and climate data) (Dial et al., 2003; Tao et al., 2004) and forest inventory data (Wulder et al., 2005). Furthermore, there is a reduction in interpreter subjective bias through the application of automated delineation methods (White et al., 2004), which may increase the consistency and reliability of mapping between different areas or dates (Wulder et al., 2006a).

Research has demonstrated that Landsat Thematic Mapper (TM) imagery can be used (along with other digital geographic data such as elevation, slope, aspect, and incoming solar radiation, where appropriate) to accurately detect mountain pine beetle red attack damage in forest stands with typical accuracies ranging from 70 to $85 \%$ (Franklin et al., 2003; Skakun et al., 2003; Coops et al., 2005; Wulder et al., 2006b). In all of these studies, the Landsat spectral data are re-projected along the principle directions of brightness, greenness, and wetness (known as a Tasseled Cap Transformation (TCT) (Kauth and Thomas, 1976; Crist and Cicone, 1984; Crist et al., 1986)) and correlated with data on forest mortality, disturbance, and anthropogenic change. Sharma and Murtha (2001) demonstrated significant differences in the TCT between lodgepole pine stands that had been attacked by mountain pine beetle and those stands that had not been attacked. Similarly, Price and Jakubauskas (1998) found that stands in Yellowstone National Park where the overstorey was progressively thinned as a result of beetle damage were distinguishable using TCT components. In addition to single scenes, a time series of Landsat imagery allows temporal changes in the TCT to be assessed and related to infestation rates. Franklin et al., (2003) used an automated 
detection algorithm (as opposed to a visual interpretation) on a $30 \mathrm{~m}$ Landsat TM image. This study detected clusters of red trees smaller than 0.2 ha with a resulting classification accuracy of $73.3 \%$ based on 360 independent mountain pine beetle field and aerial survey validation points. Skakun et al., (2003) utilized multi-temporal Landsat-7 Enhanced Thematic Mapper (ETM) imagery acquired on three separate dates. The TCT wetness index for each date was thresholded and an enhanced wetness difference index (EWDI) was used to interpret spectral patterns in stands with confirmed red-attack damage. The classification accuracy of the red-attack damage based on this type of transformation ranged from $67 \%$ to over $78 \%$ correct (Skakun et al., 2003).

At finer spatial resolutions, Kneppeck and Ahern (1989) utilized a Multi-detector Electrooptical Imaging Scanner (MEIS-II) and demonstrated that this imagery could be used to detect and map red attack damage with accuracies similar to that achieved by aerial photography. More recently, White et al. (2004) utilized an unsupervised clustering technique on 4-metre multi-spectral IKONOS imagery to detect mountain pine beetle red attack at sites with low and medium levels of attack and compared the estimates to red attack damage interpreted from aerial photography. Results indicate that within a one-pixel buffer $(4 \mathrm{~m})$ of identified damage pixels, the accuracy of red attack detection was $70.1 \%$ for areas of low infestation (stands with less than $5 \%$ of trees damaged) and $92.5 \%$ for areas of moderate infestation (stands with between $5 \%$ and $20 \%$ of trees damaged). 
The increasing availability of commercially delivered, high spatial resolution satellite data offers a potential source for the cost-effective collection of accurate, consistent, and timely data regarding mountain pine beetle impacts. A key use of this type of imagery for mountain pine beetle identification and monitoring is to complement ongoing heli-GPS surveys. The heli-GPS surveys are point surveys that typically focus on designated areas of interest, and only collect data on trees with red attack damage. Conversely, high spatial resolution remotely sensed data can fully cover an area of interest, facilitating the interpretation of both red attack and non-attack trees. In this way, the population of host trees that are at risk to attack can be fully enumerated and attack damage for a larger area of interest can be estimated. Stand-level estimates of red attack damage are necessary to estimate impact of the infestation on timber volumes.

The first objective of this research was exploratory in nature and designed to assess if high spatial resolution multi-spectral imagery (acquired by QuickBird) could be used to detect red attack damage caused by mountain pine beetle infestation. Given successful detection, a second objective was to determine the degree of correspondence between the number of QuickBird pixels identified as red attack with the number of red attack crowns observed using conventional forest health survey methods (heli-GPS). Finally, as a time series of Landsat imagery are currently being utilized to provide broad assessments of mountain pine beetle red attack damage, the correspondence between the number of red attack pixels, as identified by the high resolution imagery, and the 
variation in the EWDI was assessed in order to determine the capacity of high resolution imagery to provide validation of ongoing regional mountain pine beetle mapping.

\section{SITE DESCRIPTION}

The focus for this research is an area within Tree Farm License (TFL) 48, managed by Canadian Forest Products Ltd. TFL 48 covers approximately 650,000 ha and is located in north-eastern British Columbia near Chetwynd $\left(55.70^{\circ} \mathrm{N},-121.63^{\circ} \mathrm{W}\right)$ (Figure 1). The primary forest types within the region include high elevation forests dominated by Engelmann spruce (Picea engelmanii) and subalpine fir (Abies lasiocarpa Nutt.), midslope forests dominated by hybrid spruce (Picea hybrids), lodgepole pine, and aspen (Populus tremuloides), and the boreal foothills and plains dominated by mixed stands of white spruce (Picea glauca), aspen, and lodgepole pine (Seely et al., 2004). Lodgepole pine is a pioneer species (early seral), which tends to grow in pure even-aged stands (Koch, 1996). Within the study area, stands that are 100\% lodgepole pine have a mean age of 87 years and mean height of $15.3 \mathrm{~m}$. 


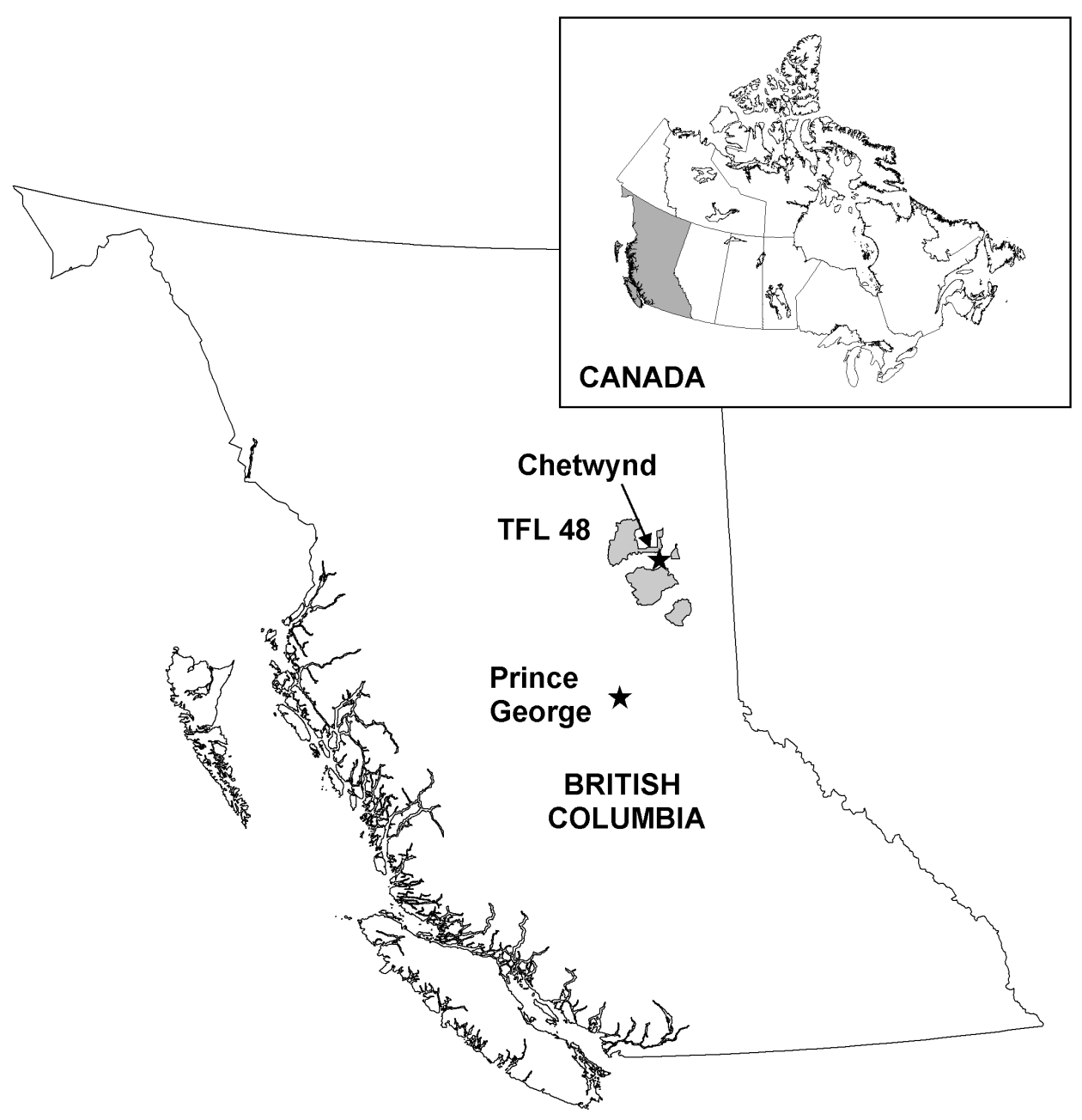

Figure 1: Location of the TFL 48 study area. 


\section{DATA}

\subsection{High Spatial Resolution Satellite Imagery}

Geocorrected QuickBird imagery was acquired on May 29 2005, under clear sky conditions, over a $6 \times 6 \mathrm{~km}$ area within TFL 48. The sun elevation at time of capture was close to optimal $\left(55.9^{\circ}\right)$ with a close-to-nadir view angle $\left(11.2^{\circ}\right)$. The imagery was geocorrected by the satellite data providers with an estimated published spatial accuracy of $14 \mathrm{~m}$ root mean square error (RMSE). Ortho-rectification was not undertaken due to the lack of availability of a high spatial resolution DEM $(<10 \mathrm{~m})$. The imagery contains four multi-spectral bands with a $2.5 \mathrm{~m}$ spatial resolution: $0.45-0.52 \mu \mathrm{m}$ (blue); $0.52-0.60 \mu \mathrm{m}$ (green); $0.63-0.69 \mu \mathrm{m}$ (red); $0.76-0.90 \mu \mathrm{m}$ (near infra-red). The QuickBird also has a panchromatic band $(0.45-0.90 \mu \mathrm{m})$ with a $0.68 \mathrm{~m}$ spatial resolution (Birk et al., 2003).

\subsection{Medium Spatial Resolution Imagery}

A time-series of Landsat imagery was available that corresponded to the onset of the mountain pine beetle infestation in the region. The pre-infestation image was Landsat-7 ETM+, acquired August 16 2001, and the post-infestation image was a Landsat-5 TM image, acquired July 29 2003. Both of these images were Path 49, Row 21. The two images were orthorectified to a UTM NAD 83 projection using a $25 \mathrm{~m}$ Digital Elevation Model (DEM) (British Columbia Ministry of Environment, Lands and Parks, 1992a) with a resulting root mean square error of less than 0.5 pixel (using a third-order polynomial transformation and cubic convolution resampling algorithm (Coops et al., 2005)). A topof-atmosphere correction was applied to both images to correct for the appropriate gain and offsets unique to each of the sensors, and a sun angle correction was undertaken 
to radiometrically correct the images to absolute reflectance (Markham and Barker, 1986).

\subsection{Forest Inventory Database}

In 2001, 1:40,000 aerial photography was flown over the area, and interpreters delineated forest stands and estimated a range of forest inventory parameters, with the resulting vectors and attributes being digitized according to Vegetation Resources Inventory (VRI) standards (British Columbia Ministry of Sustainable Resource Management, 2002a). Each forest stand was delineated as a separate polygon and labeled with a unique identification number and a series of forest attributes such as species composition (up to six species were recorded for each stand), age (in years), site index (defined as the height of an average tree at 50 years which has been allowed to grow to its full potential), and canopy closure (in 5\% intervals) (British Columbia Ministry of Sustainable Resource Management, 2002a). No damage from the mountain pine beetle infestation was present at the time the photography used for the inventory was flown in 2001. In addition to the VRI polygon coverage, plot data collected as part of the VRI validation was available. From this database 98 plots located throughout TFL 48, contained lodgepole. The mean crown radii of all lodgepole pine crowns was $1.7 \mathrm{~m}$ and a standard deviation of $0.2 \mathrm{~m}$ which is similar to other stands of similar age in the Province (Koch, 1996; Gill et al., 2000), hence, a mean crown radius of $2 \mathrm{~m}$ was assumed for the lodgepole pine in the study area. This estimate of crown size facilitated the comparison between number of QuickBird pixels and number of identified red attack tree crowns. 


\subsection{Forest Health Data}

The infestation of mountain pine beetle in TFL 48 commenced in 2001 and by 2004, the area had a wide range of infestation intensities, from stands with no attack, to those stands with light, moderate, and severe levels of infestation. Heli-GPS surveys were conducted from March to June 2004. The helicopter, flying at an altitude 500-1000 m above the forest canopy, is positioned over a cluster of red attack trees and GPS location information is collected at the centroid of the infestation cluster. The size of the clusters may vary; however, an average size is considered to have a diameter of $100 \mathrm{~m}$, with a maximum cluster diameter of $200 \mathrm{~m}$. Cluster area, shape, and compactness are not recorded (Nelson et al., 2004). At each identified location the number of attacked trees were recorded in three classes: red attack (trees with red crowns), gray-attack (dead trees with no needles), and yellow/green (trees in the process of changing color from green to red, otherwise known as faders). Given the recent nature of the infestation with the study area (3 years), the majority of infested trees were classified as either red attack or faders. A total of 40 heli-GPS survey locations, representing approximately 312 red attack trees were located on the QuickBird satellite image. The full heli-GPS dataset was randomly stratified into a calibration data set for model development (66\% of the data) and an independent validation data set for assessing model performance $(34 \%)$ of the data. 


\section{METHODS}

\subsection{Phase 1: Detection of Red Attack Using QuickBird Multispectral Data}

The first phase of the research consisted of an exploratory analysis designed to assess if the spectral and spatial resolution of QuickBird multispectral imagery is able to detect individual or small groups of red attack trees. To do this the QuickBird panchromatic image was used to facilitate the identification of individual tree crowns, or groups of crowns; and four crown classes were established: non-attack crowns, red attack crowns, fader crowns, and shadowed crowns. Whilst these four crown classes do not cover all the expected spectral variability in the scene (Peddle et al., 1999), they provide a graduation of spectral responses from non-attack to red attack stands (Goodwin et al., 2004) from which spectral separability can be determined. Two hundred tree crowns, consisting of 50 crowns in each of the four crown classes were identified. Red attack and fader crowns were all located within a $50 \mathrm{~m}$ radius (100 m diameter) of a heli-GPS location which is the standard diameter of each forest health heli-GPS plot. The locations of these crowns were manually identified by visually interrogating the panchromatic image, where possible, to view individual tree crowns and extracting the coincident multi-spectral pixels. In some cases, individual crowns could not be clearly delineated on the panchromatic image, so clusters of two or three crowns were identified, and the corresponding multispectral pixels extracted. As is common with most forest health surveys (Stone and Coops 2004) information on healthy (or non-attack) stands is not collected as part of the heli-GPS survey, and without this information, it is difficult to assess the level or nature of commission errors for the red attack damage class. To compensate for this lack of information on where healthy crowns and stands 
are located on the imagery, non-attack and shadow crowns were selected by using the panchromatic imagery, in conjunction with the Normalized Difference Vegetation Index (NDVI) (Rouse et al., 1973) values generated from the QuickBird multi-spectral bands. It was assumed that high NDVI values would be associated with increased photosynthetic activity and would therefore serve as a useful proxy for identifying non-attack trees (Wulder et al., 2006b). Healthy crowns, therefore, were selected randomly throughout the aerial swath of the forest health survey, but outside a $50 \mathrm{~m}$ buffer of any observed red attack stands, with the assumption that they were observed by the forest heath professionals and considered to show no visible signs of attack.

The spectral values for the crown samples were extracted for each of the four QuickBird multispectral bands. In addition, the NDVI and the ratio of the red $(0.63-0.69 \mu \mathrm{m})$ to green $(0.52-0.60 \mu \mathrm{m})$ wavelengths were also computed (hereafter referred to as the Red Green Index or RGI). The RGI is a potential discriminator of mountain pine beetle damage, since it emphasizes the spectral change in foliage color from green to red. Analysis of variance (ANOVA) comparisons were then used to establish which of the spectral bands or indices could discriminate each crown class (Winer, 1962; Hays 1988).

4.2 Phase 2: Correspondence of QuickBird and Heli-GPS Estimates of Red-Attack Damage

The spectral variability of each class, for each spectral wavelength and index, was examined and a simple threshold was calculated to classify the image into red attack or 
non attack. The threshold values for the RGI and the NDVI were established through an iterative process, whereby calibration points of known red-attack and nonattack locations were used to identify potential threshold values and a reserved set of independent validation points were used to assess the suitability of the threshold. This iterative process continued until errors of omission and commission were minimized. These threshold values were then applied to the QuickBird image and resulted in a binary classification of attacked and non-attacked classes. At each of the heli-GPS survey locations, the number of QuickBird pixels classified as red attack that were located within a $50 \mathrm{~m}$ buffer surrounding the location of the plot were compared to the number of red attack trees recorded by the heli-GPS survey. A simple regression approach was used to relate the number of classified pixels with the number of red attack trees, with both linear and non-linear (logarithmic) transformations of the data considered. The best model was assessed based on its standard error, significance, and coefficient of determination $\left(r^{2}\right)$. Once the model was developed, it was applied to the remaining $34 \%$ of the data (the independent validation data) and the number of red attack trees was estimated. This was then compared to the actual number of red attack trees recorded by the forest health survey in order to provide an independent assessment of the model performance.

In addition to a plot-based comparison, relationships were also investigated at the forest inventory stand (VRI polygon) scale. A polygon decomposition approach was applied to generalize the QuickBird and heli-GPS estimates to the inventory polygons (Wulder and Franklin, 2001; Wulder et al., 2005). Within each VRI polygon, the total number of 
attacked crowns from the heli-GPS and the total number of classified $2.5 \mathrm{~m}$ QuickBird pixels were compared. At the polygon scale, any positional errors with respect to the location of the heli-GPS point, as well as errors associated with the geometric processing of the QuickBird imagery will be minimized, assuming plot locations are not placed exactly along VRI boundaries. As a result of the generalization of the polygon decomposition process, it was expected that the relationship between the number of attacked trees identified from the QuickBird imagery and the number of attacked trees recorded in the forest health survey would increase in strength.

\subsection{Phase 3: Correspondence of QuickBird and Landsat Estimates of Red Attack}

\section{Damage}

Given the development of a significant model relating the number of classified attacked pixels to the number of red attack crowns, the number of QuickBird pixels classified as red attack was compared with the mean EWDI as derived from the coarser spatial resolution Landsat imagery. A similar polygon decomposition approach to that described in Phase 2 was applied. Following the procedure of Skakun et al. (2003), a TCT was generated from each of the calibrated Landsat TM and ETM+ images, resulting in a brightness, greenness, and wetness output for each image date. The EWDI was calculated by subtracting the 2003 wetness image (post-infestation) from the 2001 wetness image (pre-infestation). Positive EWDI values represent a decrease in moisture between 2001 and 2003, and this decrease in moisture is characteristic of mountain pine beetle red attack damage (Franklin et al., 2001; Skakun et al., 2003); attacked tree crowns will be considerably drier in 2003 than in 2001, when compared to 
non-attack tree crowns. As the numbers of healthy trees within each heli-GPS plot were not recorded, and estimates of tree stocking for each VRI polygon were not interpreted from the aerial photography, the density of attack trees within each VRI polygon was unknown. In order to compensate for the effect that variable VRI polygon sizes and the presence of non-forest cover types (e.g., roads, landings) found within the VRI polygons may have on the calculation of the mean EWDI value, the VRI polygons were stratified by size for analysis purposes. Only those polygons that were found within \pm one standard deviation of the mean polygon size were considered. The mean EWDI within each VRI polygon was then calculated and compared to the total number of QuickBird pixels classified as red-attack.

This analysis is undertaken to emphasize one of the key strengths of the QuickBird imagery as compared to the heli-GPS data: currently, model development and validation of Landsat derived estimates of mountain pine beetle red attack damage are hampered by the lack of information regarding stands or trees that have not been attacked. QuickBird imagery can be used to discriminate between red attack and nonattack pixels, and subsequently, to estimate the number of red attack and non-attack crowns. In this way, samples of QuickBird imagery could be used to generate information on the population-at-risk to attack, thereby facilitating the development of landscape level models of mountain pine beetle population dynamics and enhancing the accuracy of synoptic assessments of red attack damage generated from medium spatial resolution remotely sensed data such as Landsat. 


\subsection{Phase 4: Estimating Volume Losses Resulting from Mountain Pine Beetle Attack}

Estimates of the population-at-risk at the forest stand level, in conjunction with knowledge of the number of damaged trees within the stand, facilitate estimates of volume losses resulting from a successful mountain pine beetle attack. Such valueadded information products can enhance management, modeling, and monitoring activities. To demonstrate this, the number of QuickBird red attack pixels within each VRI polygon was summed, and converted to hectares of red attack (Wulder et al., 2006c). Estimates of net volume (gross volume less decay, waste, and breakage) are provided within each VRI polygon $\left(\mathrm{m}^{3}\right.$ per hectare). These volume estimates are derived using species, height, and diameter as predictor variables in a taper equation, and then calibrated using known volume loss factors. The volume estimates for lodgepole pine can then be multiplied by the amount of red attack area estimated from the QuickBird to generate an approximation of the amount of volume of lodgepole pine within the stand that has been impacted by the mountain pine beetle.

\section{RESULTS}

A true color subset of the QuickBird imagery, with the heli-GPS survey locations and the inventory polygons draped over the imagery, is shown in Figure 2. In some locations, larger areas of red attack damage were discernable from the imagery. 


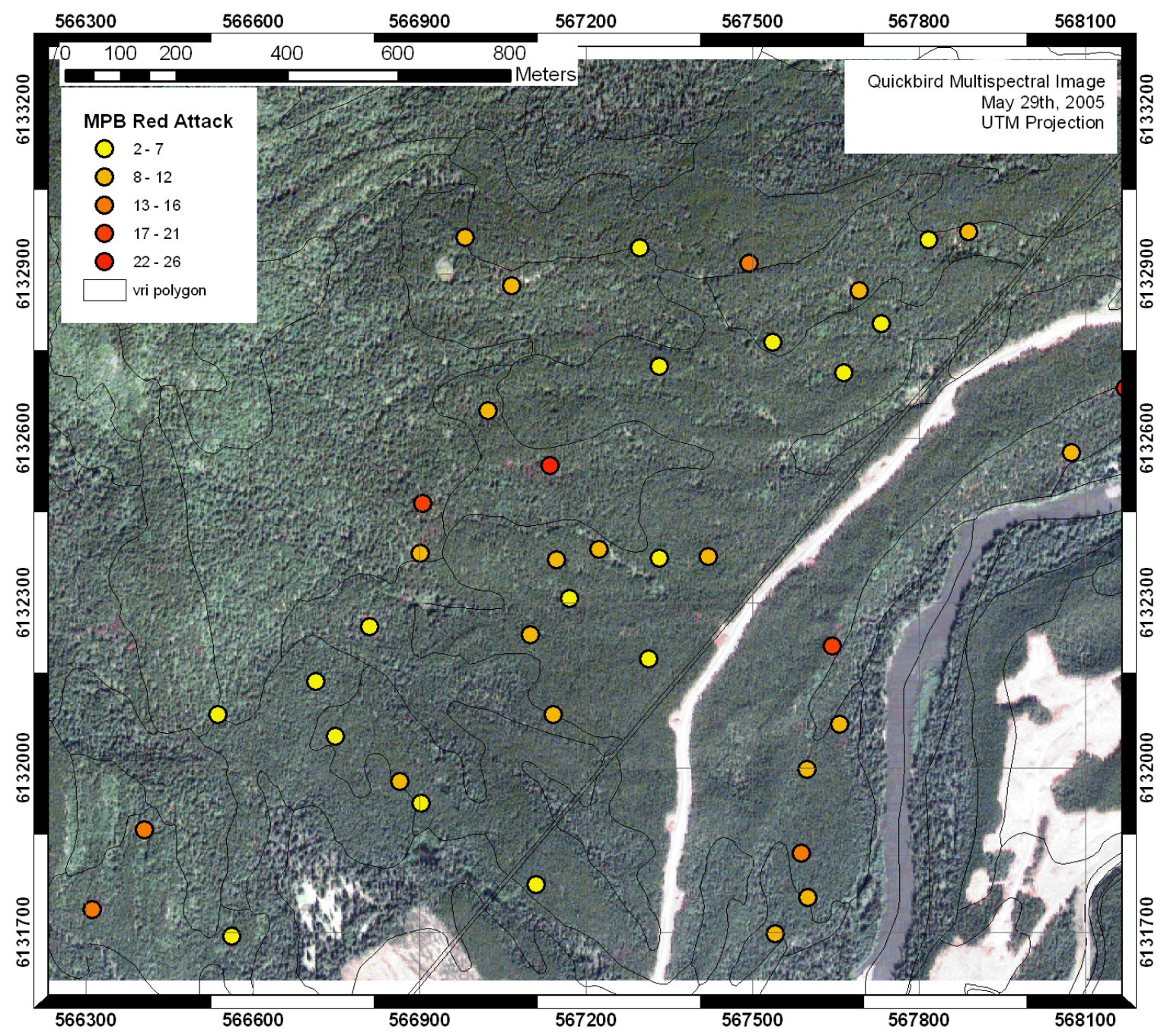

Figure 2: A sub-sample of the QuickBird imagery shown with the VRI polygons and the heli-GPS survey points, color coded according to the number of red attack trees associated with each survey point.

While mountain pine beetle surveys are typically focused on discriminating red attack trees from non-attack trees, this exploratory analysis was designed to consider the impact that two additional classes would have on the success with which red attack damage could be detected. Faders were included since they were explicitly identified in the heli-GPS survey, while shadowed crowns were included due to their dominance in the image. The mean and standard deviations of the four crown classes (fader, red- 
attack, non-attack, and shadow) indicate that there is some clear spectral distinction between the crown classes in both the raw data and the spectral indices; however, the degree of separation is highly wavelength dependent (Figure 3). The results demonstrate that at the $0.45-0.52 \mu \mathrm{m}$ (blue) wavelengths there is limited ability to discriminate between the four crown classes, with only fader crowns being discriminated from the remaining crown classes. In the $0.52-0.60 \mu \mathrm{m}$ (green) region of the spectrum, shadowed crowns are easily differentiable from the other classes. By contrast, the nonattack and red attack crowns appear similar in the $0.63-0.69 \mu \mathrm{m}$ (red) region, with the faders and the shadowed crowns being spectrally distinct at these wavelengths. In the $0.76-0.90 \mu \mathrm{m}$ (near infra-red) wavelengths, as expected, the non-attack crowns exhibit the highest reflectance, with an overall decrease in reflectance associated with increasing infestation, although there is marked overlap between classes. The NDVI demonstrates some of the difficulty in detecting a range of attack classes, with similarity between the faders and red attack crowns. In addition, there is considerable overlap between the faders, red attack, and shadowed crowns. In contrast, the RGI discriminates red attack and fader crowns from non-attack and shadowed crowns. Some overlap is found between the fader and red attack crowns, suggesting that these varying degrees of attack damage may be difficult to discriminate from one another. 


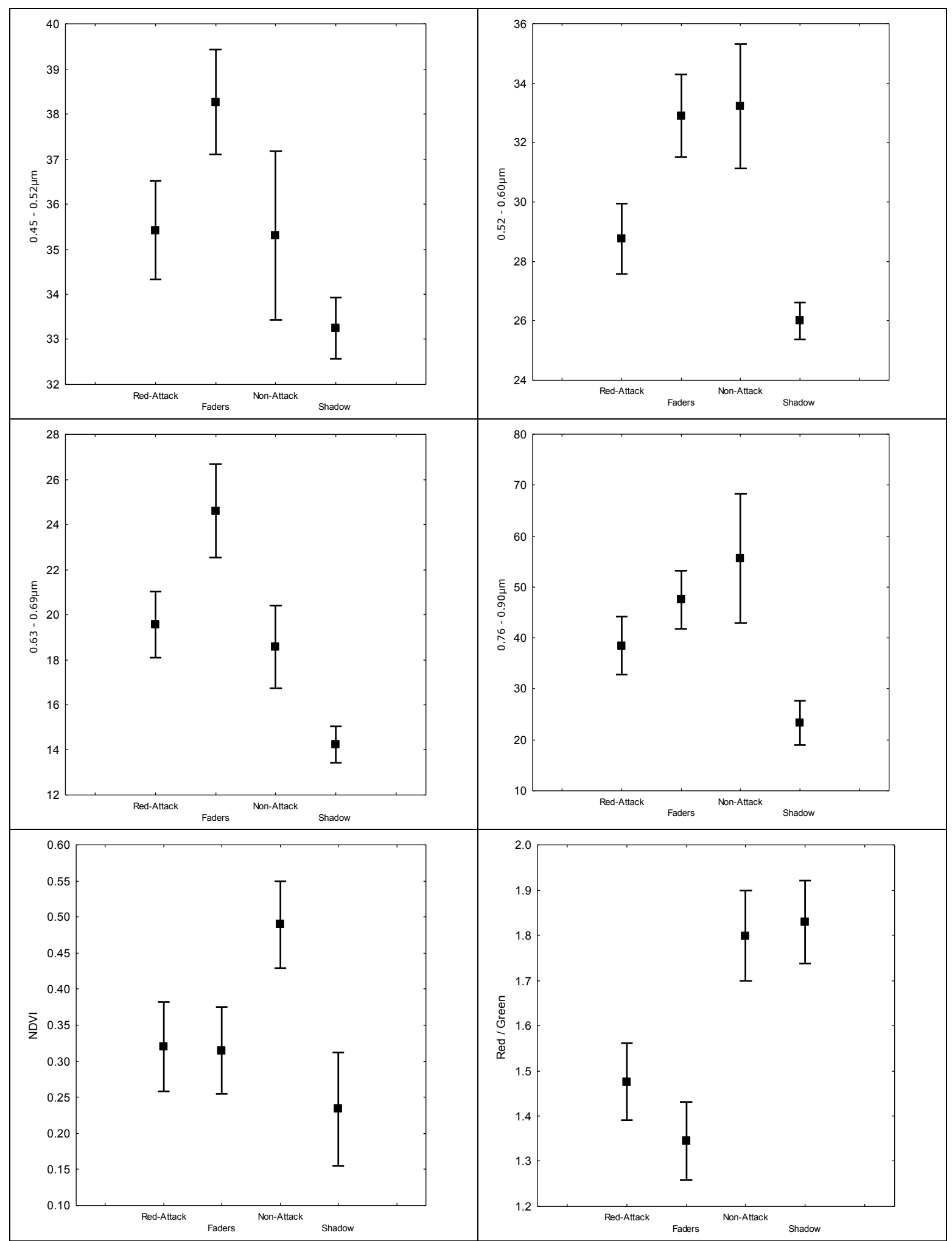

Figure 3: : Mean and standard deviations for the four QuickBird spectral wavelengths and two additional spectral indices (Normalized Difference Vegetation Index (NDVI) and the Red / Green Index (RGI)) for red attack, faders, non-attack and shadowed crowns delineated on the imagery. 
ANOVA comparisons match the general trends in Figure 3 and indicate that the 0.45 $0.52 \mu \mathrm{m}$ (blue) and $0.63-0.69 \mu \mathrm{m}$ (red) wavelengths are unable to discriminate $(p<$ 0.05 ) between non-attack and red attack crowns. The $0.52-0.60 \mu \mathrm{m}$ (green) and 0.76 $0.90 \mu \mathrm{m}$ (near infrared) regions are unable to discriminate non-attack crowns from faders. In contrast, the NDVI discriminates the fader and red attack from the non-attack, but cannot significantly separate the red attack from the fader crowns. The RGI can statistically separate fader and red attack from non-attack, but cannot discriminate between non-attack and shadowed crowns. Spectral thresholds, for both the NDVI and the RGI, were then developed based on the results shown in Figure 3, and a simple classification was undertaken on both the NDVI and the RGI images to produce binary classifications of red attack (incorporating the red attack and fader tree crown classes) and non-attack (incorporating the non-attack and shadowed crowns) classes (Figure 4).

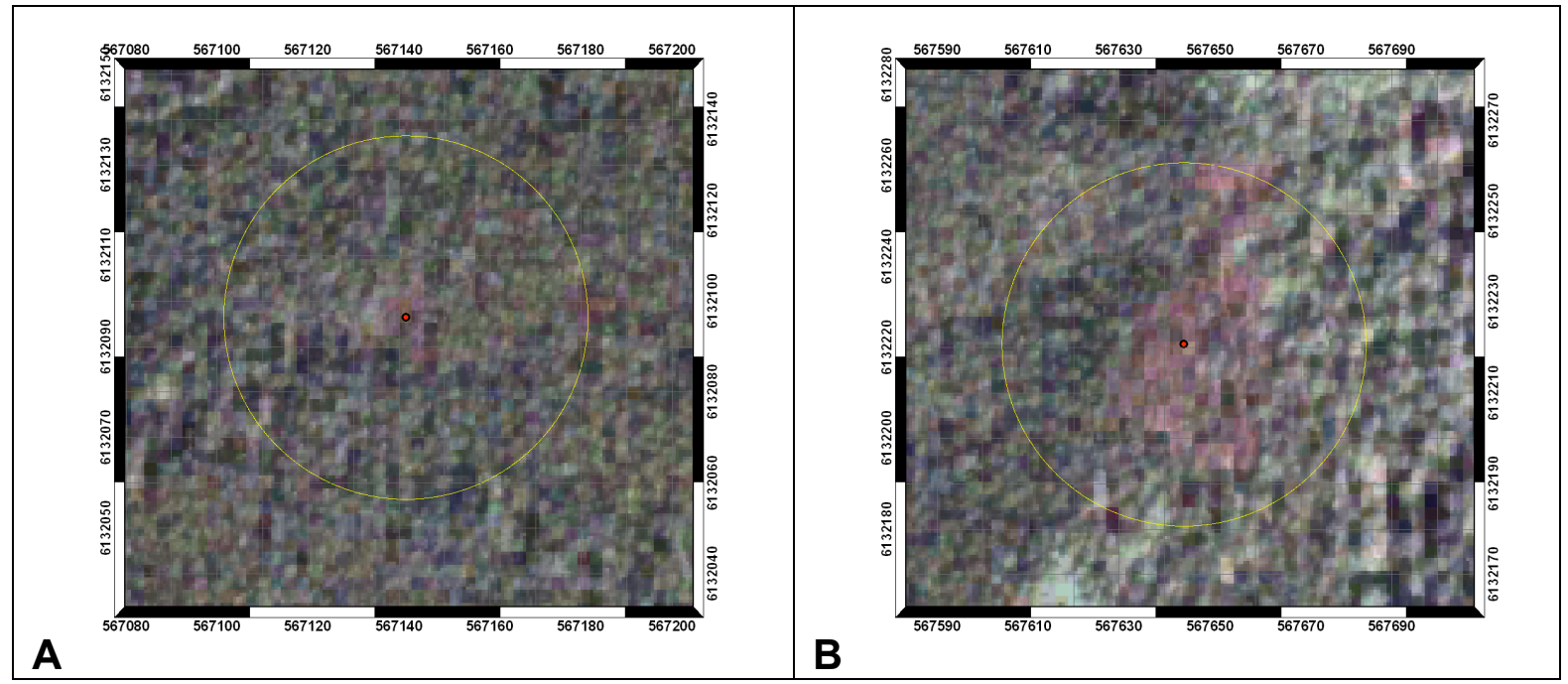




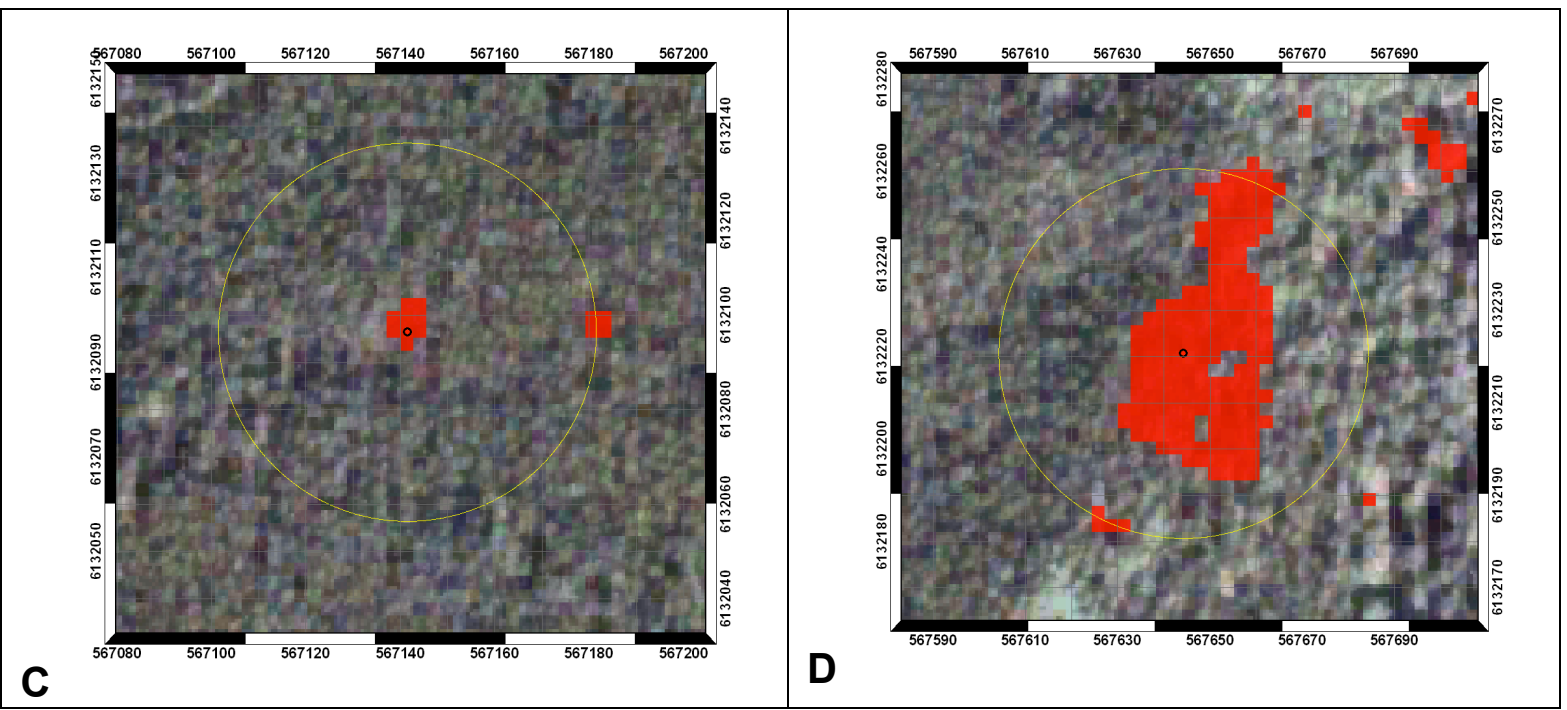

Figure 4: Graphical representation of the QuickBird (true color composite), the heli-GPS survey point, the 50 $m$ buffer surrounding the point, and the red attack pixels identified using a threshold value from the RGI. The site on the left was considered lightly infested ( $A$ and $C$ ), while the site on the right was considered heavily infested (B and D).

In both cases, a positive relationship between the number of QuickBird pixels classified as red attack and the number of observed red attack crowns were found using the model development dataset. For the NDVI (Figure 5a) the relationship is significant $(p<$ 0.001) with an $r^{2}=0.44$ and a standard error of 3.8 crowns. For the RGI (Figure $5 b$ ), the model is also significant $(p<0.001)$ with an $r^{2}=0.51$ and a standard error of 3.0 crowns (11\%). Both figures show increasing variation in the number of classified red attack pixels as the number of red attack trees recorded in the survey increases. If we assume that the average crown diameter in the study area is approximately $4 \mathrm{~m}$, then four QuickBird pixels are required to represent a single crown. Based on the increased $r^{2}$ and lower standard error the RGI model was chosen as the most appropriate and applied to the reserved validation heli-GPS survey points and the relationship between the numbers of predicted red attack pixels and the observed red attack crowns were significant $\left(r^{2}=0.48, p<0.001\right.$ with a standard error of 2.8 crowns $)$. 
A

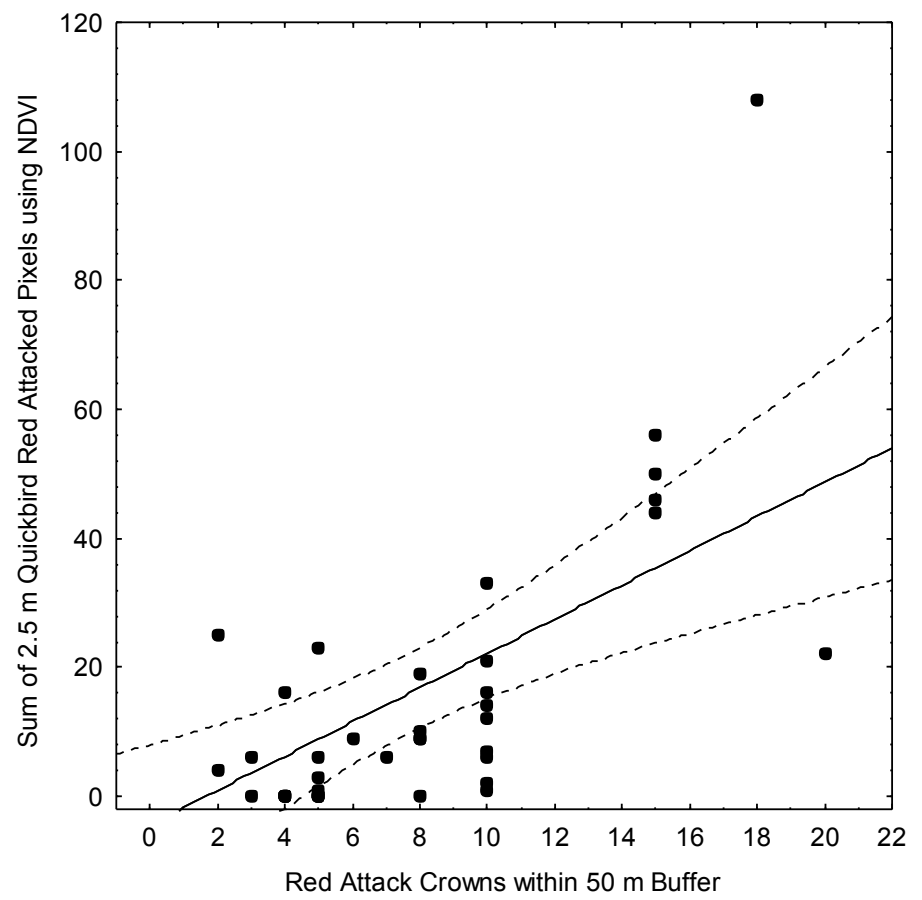

B

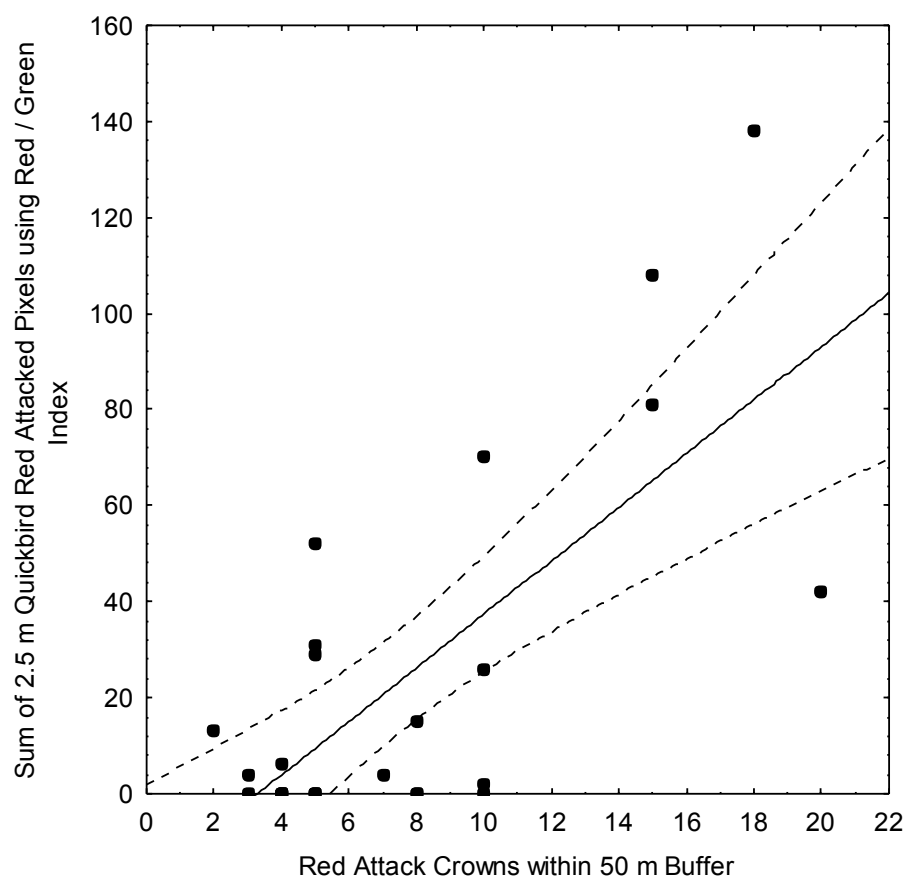

Figure 5: Relationship between number of pixels identified as red attack using the (a) NDVI and (b) the RGI, and number of observed red attack crowns from the heli-GPS survey. Solid line indicates modeled fit, dashed lines corresponded to $95 \%$ confidence intervals. 
Similar trends are evident at the forest inventory stand (VRI polygon) scale. For the NDVI classification (Figure 6a) the relationship between the numbers of classified pixels compared to the total number of red attack trees observed within the VRI polygon is not significant $(p>0.05)$. For the RGI (Figure $6 b)$ the model is more significant than at the plot level $(p<0.001)$ with an $r^{2}=0.56$ and a standard error of estimate of 11 crowns $(18 \%)$.

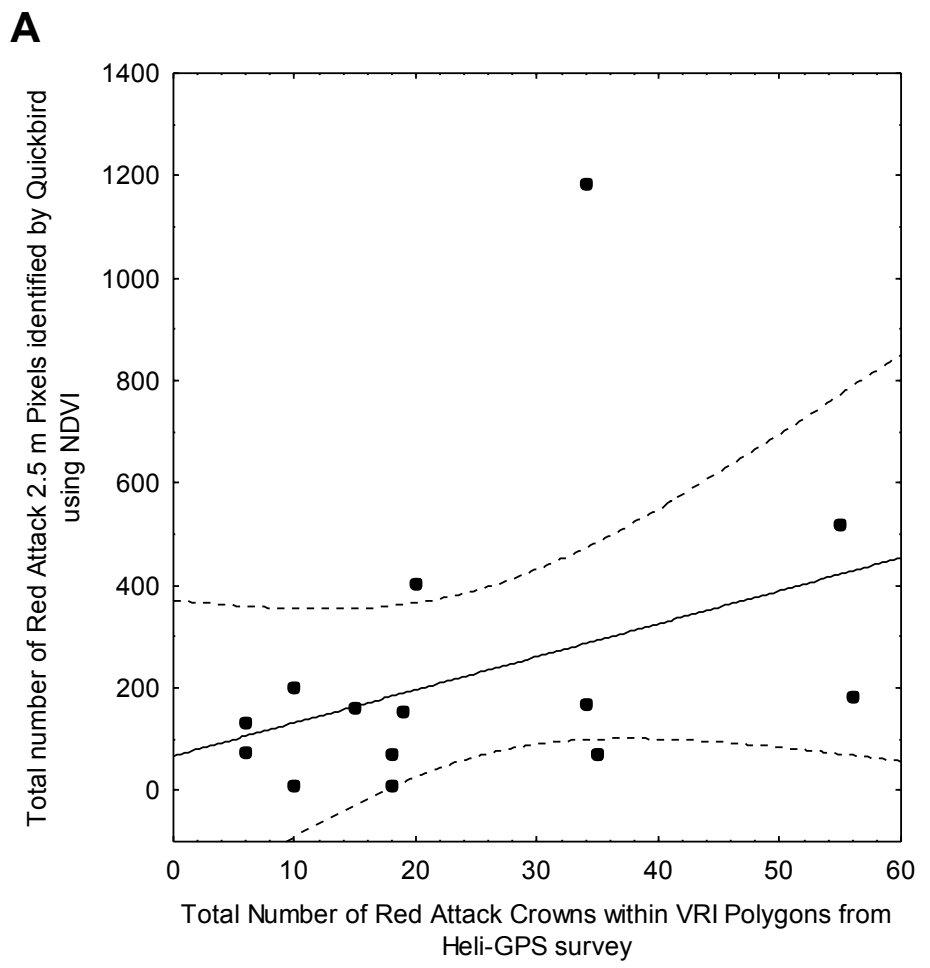




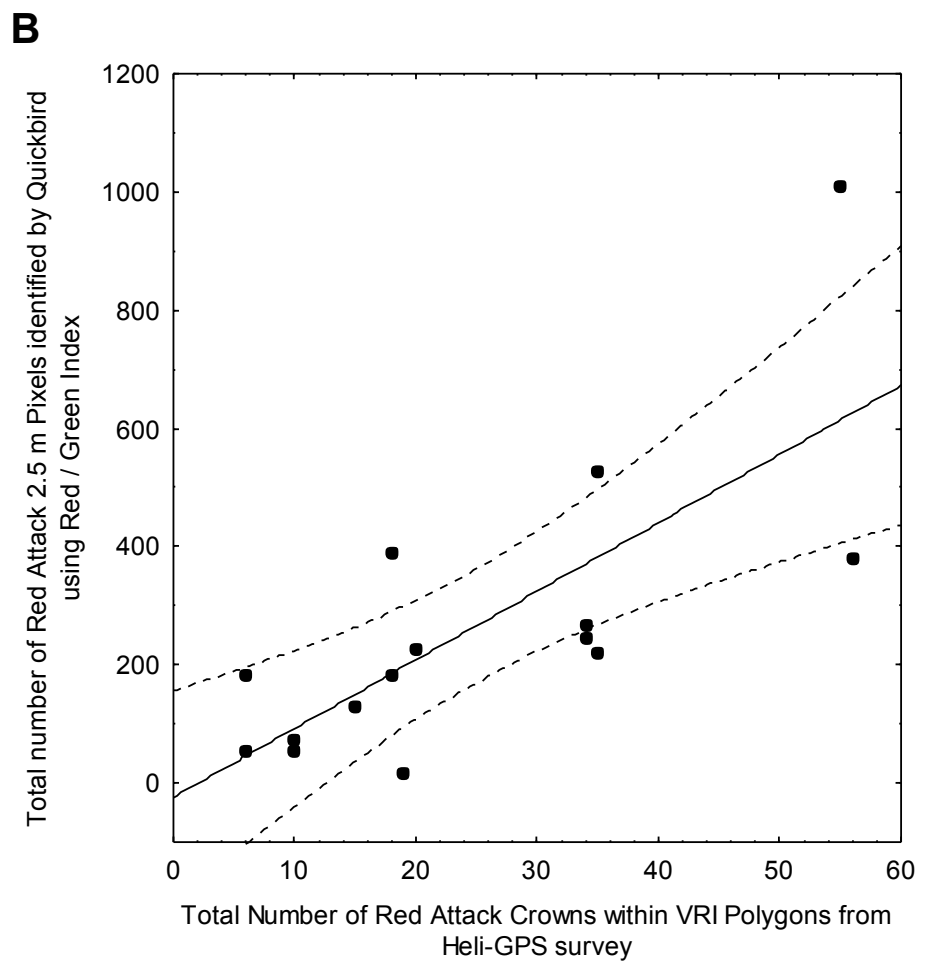

Figure 6: Counts of red attack pixels and crowns from both the QuickBird and the heli-GPS survey are decomposed to the level of the forest inventory stand (VRI polygon) for both the (a) NDVI and (b) RGI models. Solid line indicates modeled fit, dashed lines corresponded to $95 \%$ confidence intervals.

An additional application of using high spatial resolution imagery to predict the occurrence of red attack damage caused by mountain pine beetle is the ability to refine and corroborate estimates of red attack damage at the landscape level that have been derived from Landsat TM or ETM+ imagery. In this study area, the relationship between the QuickBird and Landsat estimates of red attack damage is significant using a natural logarithm transformation $\left(r^{2}=0.61, p<0.001\right.$, standard error $=1.3$ crowns $)$. The results confirm that as the total number of QuickBird pixels classified as red attack increases, so to does the magnitude of the EWDI (indicative of drier foliage) with each VRI polygon. The variation in the EWDI as a function of the number of QuickBird classified pixels indicates that VRI polygons with low levels of red attack damage have greater 
variability in the range of EWDI values; however as the level of attack increases, the variability in EWDI values decreases (Figure 7).

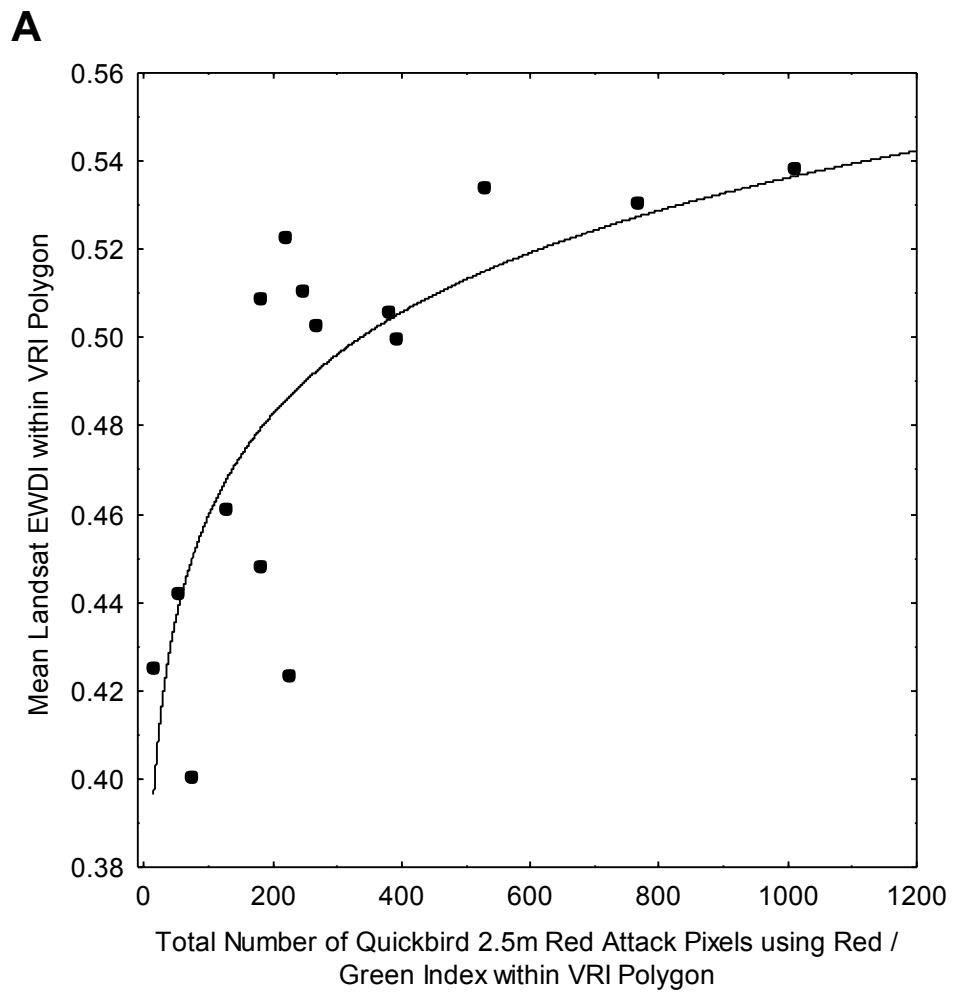

Figure 7: Graph of mean VRI EWDI against the total number of red attack trees identified by $2.5 \mathrm{~m}$ QuickBird pixels classified using the RGI. Fit is based on a natural logarithmic transformation. In order to account for variation in polygon size, only those polygons within \pm one standard deviation of the mean polygon size were analyzed.

Finally, the total number of QuickBird pixels classified as red attack were summed within each VRI polygon, and converted from pixels to hectares $(1 \mathrm{ha}=100 \times 100 \mathrm{~m})$ resulting in an area estimate of within-polygon red attack damage. This area estimate was then multiplied by a within polygon volume estimate (in $\mathrm{m}^{3} / \mathrm{ha}$ ) for lodgepole pine. Results indicate that $3555 \mathrm{~m}^{3}$ of timber within the study area have been impacted by mountain pine beetle red attack damage, which is approximately $10 \%$ of the total volume within the infested VRI polygons. Since the beetle population was just beginning to establish itself in TFL 48 at the time the data for this study was collected, these low 
volume impacts reflect the sparse and heterogeneous nature of the attack damage at this stage. The volume impacts in this area are likely to increase, as they have in other areas of British Columbia, providing favorable climactic conditions and host availability allow the beetle population to continue its expansion.

\section{DISCUSSION}

Although the exploratory analysis indicated that both the NDVI and the RGI could discriminate between red attack and non-attack, the latter generated a more significant model. The success of the RGI for discriminating red attack, compared to the results achieved using the NDVI, can potentially be explained by the underlying physiological response of the crown to mountain pine beetle attack. As the foliage fades from green to yellow to red over the spring and summer following attack, the foliage gradually desiccates and the pigments breakdown. Initially the green chlorophyll pigments are lost, then the yellow carotenes and red anthocyanins (Hill et al., 1967), and progressively the needles drop until the tree is completely defoliated. Given the rapid response of the foliage to attack, decreases in near infrared scattering (the NDVI focuses on the NIR and red spectral wavelengths) have likely already occurred by the following growing season, thus making the ratio of red to green reflectance a better discriminator of damage as it focuses on the transition from non-attack to red attack stage. The utility of the RGI is similar to the conclusions of Ahern (1988) who identified 525-565 $\mathrm{nm}$ (green) as a region of the spectrum with sensitivity to the infestation. 
The trends and variability observed in the relationship between the number of red attack pixels estimated by the multi-spectral QuickBird imagery, the number of crowns observed from the heli-GPS survey points, and the estimates of red attack damage derived from the Landsat EWDI methods, provide some insight into the potential for these technologies to assess crown condition. The relationship between the number of red attack crowns identified from the heli-GPS survey and the number of identified damaged QuickBird pixels increases in variability as the number of crowns increases. In addition, it is clear from Figure 5, that the heli-GPS estimates of red attack crowns are not actually continuous, with low levels of crown infestation $(<10$ crowns in each cluster) resulting in all crowns being individually counted. As the level of infestation increases, the interpreter is no longer able to fully enumerate the total number of crowns and instead estimates counts at 15 and 20 trees. Once stands are classified as heavily damaged (defined as more than $25 \%$ of the stand infested), individual red attack crowns are no longer enumerated by the survey technician. Harris and Dawson (1979) noted a similar "saturation effect" amongst interpreters conducting aerial overview surveys. These aforementioned issues partly explain the variation in the number of pixels identified by QuickBird compared to the heli-GPS survey at these high levels of attack, and indicate that one benefit associated with using the high spatial resolution imagery is the capability to continue to count the number of red attack pixels as damage levels progressively increase over time. 
After polygon decomposition, the relationships between QuickBird and heli-GPS estimates of red attack at the VRI polygon level follow similar trends to those shown in Figure 5, with increased variation in the QuickBird estimates as the number of red attack crowns identified by the heli-GPS survey increases. In both of these assessments, it is assumed that the relationship between the size of the individual tree crowns remains constant with the pixel size of the QuickBird imagery. Given the evenaged nature of the lodgepole pine stands we believe this to be a reasonable assumption for this application. It does however limit the transferability of the developed models between different images and forest stand types.

The incorporation of high spatial resolution imagery into forest health assessment surveys offers a number of advantages to existing programs. Heli-GPS surveys, although considered the benchmark for detailed aerial survey in British Columbia, contain errors associated with the mapped attribute (e.g., number of red attack trees) as well as positional error, due to flying height and speed. For example, at a flying elevation of $500 \mathrm{~m}$ with a view angle of 5 degrees, a location error of up to $45 \mathrm{~m}$ is possible (Wulder et al., 2004). In addition, infestation magnitude can be difficult for an observer to estimate from the air due to aircraft speed, movement, weather conditions, and surveyor experience (Wulder et al., 2004). Furthermore, the sampling design of the heli-GPS survey is typically not systematic, increasing the likelihood of omission error. Finally, no data is collected on the number and location of the non-attack tree crowns. 
The application of high spatial resolution imagery, such as QuickBird, can help minimize possible biases included in the initial heli-GPS data collection. The ability of a simple spectral index to classify red attack crowns can be applied as a component of a multistage sampling regime. Initial reconnaissance (and a complete census) of mountain pine beetle red attack damage could first be undertaken using high spatial resolution imagery, such as QuickBird. The imagery is then classified and areas of red attack damage identified - highlighting locations for further, more detailed survey by air or ground. Alternatively, the results of the QuickBird image analysis could be used to inform expectations of the heli-GPS survey results, by way of identifying trends in spatial and attribute accuracy. Since the heli-GPS survey is cheaper and more flexible in terms of its acquisition conditions and turnaround time, samples of QuickBird imagery could be used to calibrate the helicopter survey estimates. This synergy between the helicopter survey and the QuickBird estimates of red attack damage could be exploited by a long-term sample-based monitoring program.

A similar polygon decomposition approach was applied for the comparison between the QuickBird estimates of red attack damage and the mean Landsat EWDI within each forest stand. The relationship illustrated in Figure 7 provides some evidence of a threshold and saturation effect that the number of red attack crowns can have on the EWDI index (when considering VRI polygons of similar sizes). Figure 7 confirms that there is variation in the mean EWDI value (by polygon) at low levels of red attack damage, as detected with the RGI. This indicates the EWDI may be insensitive to minor changes in small numbers of red attack crowns; in situations where there are only a few 
red attack trees found within the Landsat $30 \mathrm{~m}$ pixel, other elements (e.g., non attack trees, shadows) will dominate the spectral response of the pixel. As the number of red attack QuickBird pixels within each $30 \mathrm{~m}$ Landsat pixel increases, the variability in the mean EWDI value (by polygon) reduces. When there are a large number of QuickBird red attack pixels, the relationship becomes asymptotic, indicating that an everincreasing number of red crowns have no significant effect on mean EWDI value. QuickBird data can provide estimates of the total number of trees within each VRI polygon, as well as estimates of the total number of trees in the polygon that have red attack damage. In this way, the QuickBird data can be complementary to the EWDI estimates by facilitating the calculation of severity and rates of damage increase within each VRI polygon. The relationship between the ratio of red attack trees to total trees within the polygon and the mean EWDI within the polygon, would likely provide a better indicator of the sensitivity of the EWDI to infestation levels, and would account for differences in the size, composition, and density of VRI polygons. This ratio of red attack to total trees could be calculated with QuickBird data, since the QuickBird can be used to estimate the total number of trees in the stand.

The strong relationship found between the number of red attack QuickBird pixels and the EWDI provides some insight into the detectability of red attack damage with medium spatial resolution remotely sensed data (e.g., Landsat TM/ETM+). These insights on detectability (i.e., number of red attack trees required to change EWDI values) have implications for the establishment of EWDI thresholds associated with red attack damage. The latter is particularly true in areas of very light infestation, where EWDI 
thresholds that are designed to be restrictive may result in omission error for red attack damage. The improved discriminatory power of the EWDI in the presence of larger numbers of trees with red attack damage provides empirical evidence for the improved detectability results associated with larger numbers of red attack trees (Skakun et al., 2003; White et al., 2004). This also suggests that samples of QuickBird data could be used to calibrate and validate broader landscape estimates of red attack damage derived from medium resolution data such as Landsat TM and ETM+.

The results presented in this paper have implications for the generation of volume losses that result from mountain pine beetle infestations. The high spatial resolution of the QuickBird imagery and the significant relationship between the number of red attack trees observed from the heli-GPS survey and the detected QuickBird pixels, suggest the imagery could provide a reliable proxy for the number of red attack crowns found within an inventory polygon (accounting for variability in crown size). Since the QuickBird data is spatially extensive, it would be better suited to this type of analysis than the nonsystematic point data collected from the heli-GPS survey. With information contained in the inventory such as species, age, and height, estimates of the total volume in the stand that is impacted by the beetle can be calculated. Finally, the ability to collect QuickBird data repeatedly over multiple time intervals, coupled with the ability to apply the same image analysis protocols, can provide a consistent and reliable means to detect and monitor red attack damage over time. 


\section{CONCLUSIONS}

The results of this research indicate that QuickBird high spatial resolution satellite imagery can be used to detect mountain pine beetle red attack damage, and that the total number of QuickBird pixels classified as red attack are significantly correlated to the number of tree crowns with red attack damage identified by a conventional heli-GPS survey. These results suggest that high spatial resolution satellite imagery offers an alternative for estimating the location and extent of red attack damage, at a similar spatial resolution to heli-GPS, where the latter is unavailable. Furthermore, high spatial resolution data can be used to augment existing forms of mountain pine beetle survey, due to the large area coverage possible with this data, and the capability to report the population-at-risk (i.e., total number of trees), as well as the total number of damaged trees. In addition to detecting and mapping red attack damage, samples of QuickBird data can have a role for calibrating and validating landscape level estimates of red attack damage such as that derived from a time series of Landsat imagery. Finally, the

stand level estimates of red attack generated from high spatial resolution imagery may be used to approximate the impact of the beetle on timber volumes within the stand. 


\section{ACKNOWLEDGMENTS}

We are grateful for comments from three anonymous reviewers whose suggestions significantly improved the manuscript. We acknowledge CANFOR for provision of the VRI polygon, and plot, forest inventory information and access to the Digital Elevation Model. This project is funded by the Government of Canada through the Mountain Pine Beetle Initiative, a six-year, $\$ 40$ million program administered by Natural Resources Canada, Canadian Forest Service. Additional information on the Mountain Pine Beetle Initiative may be found at: http://mpb.cfs.nrcan.gc.ca. 


\section{REFERENCES:}

Ahern, F.J. (1988). The effects of bark beetle stress on the foliar spectral reflectance of lodgepole pine. International Journal of Remote Sensing, 9, 1451-1468.

Aldrich, R.C., Heller, R.C., \& Bailey, W.F. (1958). Observation limits for aerial sketchmapping southern pine beetle in the southern Appalachians. Journal of Forestry, 56, 200-202.

Amman, G. D. (1982). The mountain pine beetle - identification, biology, causes of outbreaks, and entomological research needs. Proceedings of the Joint Canada/USA Workshop on Mountain Pine Beetle Related Problems in Western North America (pp. 7-12). Victoria, British Columbia, Canada: Natural Resources Canada, Canadian Forest Service, Pacific Forestry Centre.

Birk, R.J., Stanley, T., Snyder, G.I., Hennig, T.A., Fladeland, M.M., \& Policelli, F., (2003). Government programs for research and operational uses of commercial remote sensing data. Remote Sensing of Environment, 88, 3-16.

British Columbia Ministry of Environment, Lands and Parks. (1992). British Columbia Specifications and Guidelines for Geomatics, Content Series Volume 3: Digital Baseline Mapping at 1:20000, Release 2.0 January 1992. Geographic Data BC: Victoria, British Columbia, Canada.

http://srmwww.gov.bc.ca/bmgs/trim/trim/trim_specs.html

British Columbia Ministry of Forests. (1995). Bark Beetle Management Guidebook. Forest Practices Branch: Victoria, British Columbia, Canada. www.for.gov.bc.ca/tasb/legsregs/fpc/fpcguide/beetle/betletoc.htm

British Columbia Ministry of Forests. (2004). Ministry of Forests Land-Base Investment Program: Aerial Detection Standards for Bark Beetle Management. Interim for FY 2004/05. Victoria, British Columbia, Canada: Forest Practices Branch. http://www.for.gov.bc.ca/hcp/fia/landbase/AerialDetectionStandardforBarkBeetle Management.doc

British Columbia Ministry of Forests \& Canadian Forest Service. (2000). Forest Health Aerial Overview Survey Standards for British Columbia: The B.C. Ministry of Forests adaptation of the Canadian Forest Service's FHN Report 97-1 "Overview Aerial Survey Standards for British Columbia and the Yukon", Version 2.0. Victoria, British Columbia, Canada: Resources Inventory Committee. http://srmwww.gov.B.C.ca/risc/pubs/teveg/foresthealth/index.htm

British Columbia Ministry of Forests and Range. (2005). Mountain pine beetle affects 8.5 million hectares. Press Release: December 20, 2005. Victoria, British Columbia, Canada. http://www.for.gov.bc.ca/hfp/mountain_pine_beetle/2005MPBsurvey.pdf

British Columbia Ministry of Sustainable Resource Management. (2002a). Vegetation Resources Inventory: Photo Interpretation Procedures, Version 2.4.Victoria, 
British Columbia, Canada: Resources Inventory Committee. http://www.for.gov.bc.ca/hts/vri/standards/\#photo

British Columbia Ministry of Sustainable Resource Management. (2002b). Gridded Digital Elevation Model Product Specifications, Edition 2.0. Victoria, British Columbia, Canada: Base Mapping and Geomatics Services Branch. http://srmwww.gov.bc.ca/bmgs/products/BC-DEM-specifications-2002-12.pdf

Coops, N., Wulder, M.A. \& White, J.C. (2005). Characterizing preferential attack by mountain pine beetle in susceptible forest stands. Forest Ecology and Management, In review.

Crist, E. P., \& Cicone, R.C., (1984). Application of the tasseled cap concept to simulated Thematic Mapper data. Photogrammetric Engineering and Remote Sensing, 50, $327-331$.

Crist, E. P., Lauren, R., \& Cicone, R.C., (1986). Vegetation and soils information contained in transformed Thematic Mapper data. Final Proceedings: IGARSS '86 Symposium. Zurich, Switzerland, 8 - 11 September ( pp. 1465 - 1470) Noordwijk, The Netherlands: ESA Publ. Division, SP-254.

Dial, G., Bowen, H., Gerlach, F., Grodecki, J., \& Oleszczuk, R., (2003). IKONOS satellite, imagery and products. Remote Sensing of Environment, 88, 23-36.

Franklin, S. E., Lavigne, M. B., Moskal, L. M., Wulder, M. A., \& McCaffrey, T.M., (2001). Interpretation of forest harvest conditions in New Brunswick using Landsat TM enhanced wetness difference imagery (EWDI) Canadian Journal of Remote Sensing, 27, 118-128.

Franklin, S., Wulder, M., Skakun, R., \& Carroll, A. (2003). Mountain pine beetle red attack damage classification using stratified Landsat TM data in British Columbia, Canada. Photogrammetric Engineering and Remote Sensing, 69, 283-288.

Frayer, W.E., \& Furnival, G.M. (1999). Forest survey sampling designs: a history. Journal of Forestry, 97, 4-10.

Gill, S.J., Biging, G.S. \& Murphy, E.C. (2000). Modeling conifer tree crown radius and estimating canopy cover. Forest Ecology and Management, 126, 405-416.

Goodwin, N., Coops, N.C. Stone, C. (2005) Quantifying Forest Canopy Condition from Airborne Imagery using Spectral Mixture Analysis and Fractional Abundances. International Journal of Applied Earth Observation and Geoinformation, 7, 11-28

Harris, J.W.E., \& Dawson, A.F., (1979). Evaluation of aerial forest pest damage survey techniques in British Columbia. Victoria, British Columbia, Canada: Environment Canada, Canadian Forestry Service, Pacific Forest Research Centre Information Report, BC-X-198

Hays, W. L. 1988. Statistics (4th edition). New York: CBS College Publishing.

Henigman, J., Ebata, T., Allen, E., Holt, J., \& Pollard, A. (Eds.) (1999). Field guide to forest damage in British Columbia. Victoria, British Columbia, Canada: British Columbia Ministry of Forests. 
Hill, J.B., Poop, H.W., \& Grove, A.R. Jr. (1967). Botany: A textbook for colleges, 4th Edition. Toronto: McGraw-Hill Book Co.

Kauth, R. J., \& Thomas, G.S., (1976). The tasseled cap - a graphic description of spectral-temporal development of agricultural crops as seen by Landsat. Final Proceedings: 2nd International Symposium on Machine Processing of Remotely Sensed Data (pp. 4B-41-4B-51). West Lafayette, Indiana, USA: Purdue University.

Kneppeck, I.D., \& Ahern, F.J. (1989). A comparison of images from a pushbroom scanner with normal color aerial photographs for detecting scattered recent conifer mortality. Photogrammetric Engineering and Remote Sensing, 55, 333337.

Koch, P. (1996). Lodgepole pine in North America. Madison Wisconsin, USA: Forest Products Society.

Markham, B., \& Barker, J. (1986). Landsat MSS and TM post calibration dynamic ranges, exoatmospheric reflectances and at satelite temperature. EOSAT Landsat Technical Notes, 1, 3-7.

McConnell T.J., Johnson, E.W., \& Burns, B. (2000). A Guide to Conducting Aerial Sketchmapping Surveys. Fort Collins, Colorado, USA: USDA Forest Service, Forest Health Technology Enterprise Team. Report FHTET 00-001.

Murtha, P. A. (1978). Remote sensing and vegetation damage: A theory for detection and assessment. Photogrammetric Engineering Remote Sensing, 44, 11471158.

Murtha, P. A., \& Wiart, R. (1987). PC-based digital image analysis for mountain pine beetle green attack: Preliminary results. Canadian Journal of Remote Sensing , 13, 92-95.

Nelson, T., Boots, B., \& Wulder, M.A. (2004). Spatial-temporal analysis of mountain pine beetle infestations to characterize pattern, risk, and spread at the landscape level. In: Shore, T.L., Brooks, J.E., Stone, J.E. (Eds.), Mountain Pine Beetle Symposium: Challenges and Solutions, 30-31 October 2003. Kelowna, British Columbia, Canada (pp. 164-173). Victoria, British Columbia, Canada: Natural Resources Canada, Canadian Forest Service, Pacific Forestry Centre. Information Report BC-X-399.

Nelson, T., Boots, B., \& Wulder, M.A. (2006). Large Area Mountain Pine Beetle Infestations: Spatial Data Representation and Accuracy. Forestry Chronicle, In press.

Peddle, D.R., Hall, F.G. \& LeDrew, E.F. (1999). Spectral mixture analysis and geometric-optical reflectance modelling of boreal forest biophysical structure. Remote Sensing of Environment, 67, 288-297.

Price, K. P., \& Jakubauskas, M.E. (1998). Spectral retrogression and insect damage in lodgepole pine successional forests. International Journal of Remote Sensing, $19,1627-1631$. 
Rouse, J.W., Haas, R. H., Shell, J. A., \& Deering, D. W. (1973). Monitoring vegetation systems in the Great Plains with ERTS-1. In 3rd Earth Resources Technology Satellite Symposium, 1, 309-317.

Safranyik, L., Shrimpton, D., \& Whitney, H.S. (1974). Management of lodgepole pine to reduce losses from the mountain pine beetle. Victoria, British Columbia, Canada: Environment Canada, Canadian Forest Service, Pacific Forest Research Centre.

Seely, B., Nelson, J., Wells, R., Peter, B., Meitner, M., Anderson, A., Harshaw, H., Sheppard, S., Bunnell, F., Kimmins H., \& Harrison, D. (2004). The application of a hierarchical, decision-support system to evaluate multi-objective forest management strategies: a case study in northeastern British Columbia, Canada. Forest Ecology and Management, 199, 283-305.

Sharma, R., \& Murtha, P.A. (2001). Application of Landsat TM tasseled cap transformations in detection of mountain pine beetle infestations. Final Proceedings: 23rd Canadian Symposium of Remote Sensing, 21-24 August 2001, Quebec City, Quebec, Canada.

Skakun, R.S., Wulder, M.A., \& Franklin, S.E., (2003). Sensitivity of the Thematic Mapper Enhanced Wetness Difference Index (EWDI) to detect mountain pine needle red attack damage. Remote Sensing of Environment, 86, 433-443.

Stone, C., \& Coops, N.C. (2004) Assessment and monitoring damage from insects in Australian native forest and plantations. Australian Journal of Entomology, 43, 283-292.

Tao, C.V., Hu, Y., \& Jiang, W., (2004). Photogrammetric exploitation of IKONOS imagery for mapping applications. International Journal of Remote Sensing, 25, 2833-2853.

Unger, L., (1993). Mountain pine beetle. Forest Pest Leaflet, 76. Victoria, British Columbia, Canada: Natural Resources Canada, Canadian Forest Service, Pacific Forestry Centre.

White, J.C., Wulder, M.A., Brooks, D., Reich, R., \& Wheate, R. (2004). Mapping mountain pine beetle infestation with high spatial resolution satellite imagery. Remote Sensing Environment, 96, 240-251.

Winer, B. J. (1962). Statistical principles in experimental design. New York: McGrawHill.

Wood, S.L., (1963). A revision of the bark beetle genus Dendroctonus Erichson (Coleoptera: Scolytidae). Great Basin Naturalist, 23, 1-117.

Wood, C., \& Unger, L. (1996). Mountain pine beetle. A history of outbreaks in pine forests in British Columbia, 1910 to 1995. Victoria, British Columbia, Canada: Natural Resources Canada, Canadian Forest Service, Pacific Forestry Centre.

Wulder, M.A., \& Franklin, S.E. (2001) Polygon decomposition with remotely sensed data: Rationale, methods, and applications. Geomatica, 55, 11-21. 
Wulder, M.A., Dymond, C.C., \& Erickson, B. (2004). Detection and monitoring of the mountain pine beetle. Victoria, British Columbia, Canada: Natural Resources Canada, Canadian Forest Service, Pacific Forestry Centre. Information Report BC-X-398.

Wulder, M.A., Skakun, R.S., Franklin, S.E., \& White, J.C., (2005). Enhancing forest inventories with mountain pine beetle infestation information. Forestry Chronicle, 81, 149-159.

Wulder, M.A., Dymond, C.C., White, J.C., Leckie, D.G., \& Carroll, A.L. (2006a). Surveying mountain pine beetle damage of forests: A review of remote sensing opportunities. Forest Ecology and Management, 221, 27-41.

Wulder, M.A, White, J.C., Bentz, B., Alvarez, F., \& Coops, N.C. (2006b). Estimating the probability of mountain pine beetle red attack damage. Remote Sensing of Environment, In press.

Wulder, M.A., White, J.C., Bentz, B., \& Ebata, T. (2006c). Augmenting the existing survey hierarchy for mountain pine beetle red attack damage with satellite remotely sensed data. Forestry Chronicle, In press. 